\title{
Coulomb effects in a ballistic one-channel S-S-S device
}

\author{
D. A. Ivanov ${ }^{1,2}$, M. V. Feigel'man ${ }^{1}$ \\ 1 L.D.Landau Institute for Theoretical Physics, 117940 Moscow, Russia \\ 2 12-127 M.I.T. Cambridge, MA, 02139 USA
}

(November 19, 1997)

\begin{abstract}
We develop a theory of Coulomb oscillations in superconducting devices in the limit of small charging energy $E_{C} \ll \Delta$. We consider a small superconducting grain of finite capacity connected to two superconducting leads by nearly ballistic single-channel quantum point contacts. The temperature is supposed to be very low, so there are no single-particle excitations on the grain. Then the behavior of the system may be described as quantum mechanics of the superconducting phase on the island. The Josephson energy as a function of this phase has two minima which become degenerate at the phase difference on the leads equal to $\pi$, the tunneling amplitude between them being controlled by the gate voltage at the grain. We find the Josephson current and its low-frequency fluctuations and predict their periodic dependence on the induced charge $Q_{x}=C V_{g}$ with period $2 e$.
\end{abstract}

\section{INTRODUCTION.}

Coulomb effects in several different types of three-terminal devices consisting of an island connected to external leads by two weak-link contacts, and capacitatively coupled to an additional gate potential, have been extensively studied during last years. The systems with a normal-metal island and leads were studied theoretically both in the tunnel-junction limit [1] and in the case of a quantum point contact with almost perfect transmission [2]. The theory of charge-parity effects and Coulomb modulation of the Josephson current was investigated in details in [3]. All the above-mentioned systems at present are realized experimentally. Recently it was shown to be possible to produce quantum point contact between two superconductors via a normal-conductive region made of two-dimensional electron gas (2DEG) [4]; smeared step-wise behaviour of the critical current was observed, in qualitative agreement with predictions [5] for the superconductive quantum contact with a few conduction channels of high transmittivity. An observation of a non-sinusoidal current-phase relation in superconducting mechanically-controllable break junctions has been reported in [6], again in agreement with [5]. Another interesting experimental achivement was reported in [7], where S-N-S contact with a size comparable to the de Broghle wavelength in the $\mathrm{N}$ region made of $\mathrm{BiPb}$ was realized and nonmonotonic behaviour of the critical current with the thickness of normal region was found. This remarkable development of technology points to the principal possibility to make a system of a small superconductive (SC) island connected to the superconductive leads by two quantum point contacts (QPC). In such a system macroscopic quantum effects due to competition between Josephson coupling energy and Coulomb (charging) energy could be realized together with quantization (due to small number of conductive channels) of the Josephson critical current.

In the present paper we develop a theory for an extreme case of such a system, namely, for the case of two almost ballistic one-channel QPCs connecting a small SC island with two SC leads. We consider the limit of the characteristic charging energy much smaller than the superconducting gap, $E_{C} \ll \Delta$, and, therefore, the Coulomb effects are small. We derive the dependences of the average Josephson current across the sytem, and its fluctuations (noise power) as functions of the SC phase difference between the leads $\alpha$, and of the electric gate potential $V_{g}$. The Coulomb effects reveal themselves at phase differences $\alpha$ close to $\pi$, when the two lowest states are almost degenerate. We show that such a system realizes a tunable quantum two-level system (pseudo-spin 1/2) which may be useful for the realization of quantum computers (see e.g. [8] 11] ).

The paper is organized as follows. We start with considering a single QPC connecting a superconducting island to a single lead (Section II). We find the oscillations of the effective capacitance on the island as a function of the gate potential (in some analogy with Matveev's results [2] for a normal QPC). Depending on the backscattering probability in the contact, it may be described either in adiabatic or in diabatic approximation. We find the condition for the diabatic-adiabatic crossover. Then in Section III we formulate a simple model for the double-contact system in the adiabatic approximation. We replace the full many-body problem by a quantum-mechanical problem for the dynamics of the SC phase on the middle island. In Sec.IV we calculate average Josephson current through the system as a function of $\alpha$ and $V_{g}$, with a particular emphasis on the case of the phase difference $\alpha$ close to $\pi$ (when our effective two-level system is almost degenerate). Sec.V is devoted to the analysis of the Josephson current noise; we calculate integrated intensity $S_{0}$ of the "zero"-frequency noise (an analogue of the noise calculated in [12 14] for a 
single superconduvtive QPC) as well as finite-frequency noise $S_{\omega}$ due to transitions between the two almost-degenerate levels. Finally, we present our conclusions in Sec.VI.

\section{ADIABATIC-DIABATIC CROSSOVER IN A SINGLE SUPERCONDUCTING QUANTUM POINT CONTACT.}

Consider a small superconducting island connected to an external superconducting lead by an one-channel nearly ballistic quantum point contact [5:15]. The electric potential of the grain may be adjusted via a gate terminal (fig. 1a). Following [5] we assume that the contact is much wider than the Fermi wavelength (so that the transport through the constriction may be treated adiabatically), but much smaller than the coherence length $\xi_{0} \equiv \hbar v_{F} / \pi \Delta$ (where $v_{F}$ is the Fermi velocity, $\Delta$ is the superconducting gap).
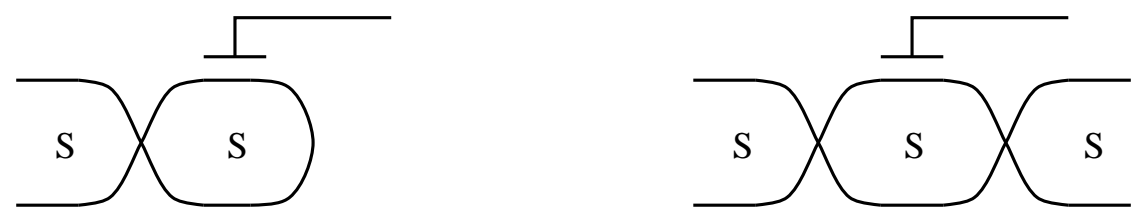

FIG. 1. (a) Single $\mathrm{QPC}$. The system consists of a SC grain connected to a SC lead via a QPC. A gate terminal is used to control the electric potential of the grain. (b) Double-contact S-S-S system. The second terminal is added to the single-QPC setup.

Our assumption of low temperature is that the average number of one-electron excitations on the island is much less than one. Then they cannot contribute to the total charge of the grain and we may restrict our Coulomb blockade problem to the evolution of the superconducting phase only. The condition of low temperature is then $T<\Delta / \log (V \nu(0) \Delta)$, where $V$ is the volume of the grain, $\nu(0)$ is the density of electron states at the Fermi level.

We neglect phase fluctuations in the bulk of the island and describe the whole island by a single superconducting phase $\chi$. At a fixed value of the phase on the island, the spectrum of the junction consists of the two Andreev states localized on the junction and the continuum spectrum above the gap $\Delta$ [15] (fig. 2). The energies of the Andreev states lie below the gap:

$$
E(\chi)= \pm \Delta \sqrt{1-t \sin ^{2}(\chi / 2)}
$$

where $\chi$ is the phase difference at the contact, $t$ is the transmission coefficient.

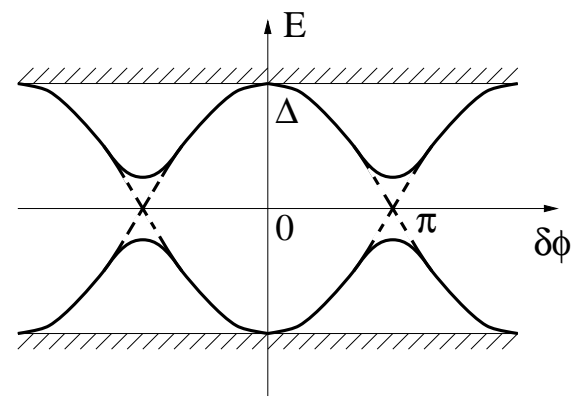

FIG. 2. Single-contact energy spectrum. The spectrum consists of the continuum of delocalized states and the two Andreev (subgap) states. Dashed lines denote Andreev states in the absence of backscattering (diabatic terms). Solid lines are the states split by backscattering (adiabatic terms).

At $t=1$, the spectrum of Andreev states (11) has a level crossing point at $\chi=\pi$. At this point, the left and right Andreev states have equal energies, but in the absence of backscattering $(t=1)$ the transitions between them are impossible. Therefore, we expect that an ideal ballistic contact cannot adiabatically follow the ground state as the phase $\chi$ changes, but remains on the same left or right Andreev state as it passes the level-crossing point $\chi=\pi$. We borrow the terminology from the theory of atomic collisions [16] and call the (crossing) Andreev levels at $t=1$ diabatic terms (dashed lines in fig. 2), and the split levels - adiabatic terms (solid lines in fig. 2). Instead of transmission coefficient $t$, it will be more convenient to speak of the reflection coefficient $r=1-t$. At $r=0$, the contact is described by diabatic terms. As $r$ increases, the transitions occur between the terms, and at sufficiently large $r$ the 
system will mostly adiabatically follow the split Andreev levels. In this section we study adiabatic-diabatic crossover and find the crossover scale for the reflection coefficient $r$.

We assume that the reflection probability $r \ll 1$ (almost unity transmission) and that the charging energy $E_{C} \ll \Delta$ (the charging energy is defined by $E_{C}=(2 e)^{2} / C$ ). The latter assumption appears natural, because, like in the tunnel junctions [17] we expect that the capacitance $C$ of the grain has an additional contribution from the capacitance of the point contact. This capacitance is of order $\Delta / e^{2}$. A more detailed discussion of this phenomenon will be given elsewhere. At the moment we just mention that this contribution to the capacitance leads to the inequality $E_{C} \leq \Delta$.

To probe the degree of adiabaticity, we study the periodic dependence of the ground state energy $E_{0}$ on the gate voltage. Because of the weakness of charging effects, this dependence will be sinusoidal:

$$
E_{0}\left(V_{g}\right)=\varepsilon \cos (2 \pi N)
$$

(where $N=V_{g} C / 2 e$ is the dimensionless voltage), and we are interested in the amplitude $\varepsilon$ of these oscillations. The physical meaning of this periodicity is the oscillations of the induced charge on the grain - it follows immediately from the relation

$$
\delta Q=\frac{C}{2 e} \frac{\partial E_{0}}{\partial N} .
$$

There is a simple physical explanation of the sinusoidal dependence (2). The ground-state energy modulation is determined by phase-slip processes in the contact. Such processes are phase tunneling events with phase changing by $\pm 2 \pi$. While the magnitudes of the clockwise and counter-clockwise tunneling amplitudes are the same, their phases are $\pm 2 \pi N$. This results in the expression (2). Higher-order tunneling processes would give rise to higher-order harmonics in the periodic $N$-dependence. This argument shows that the amplitude of oscillations $\varepsilon$ coincides with the phase-tunneling amplitude and, therefore, provides a good measure of adiabaticity in the phase dynamics.

Under assumption $E_{C} \ll \Delta$, we may describe the contact by the dynamics of the phase on the grain and thus reduce the problem to a single-particle quantum mechanics. Since we restrict our attention to low lying excitations, it is only necessary to include the two Andreev levels on the junction. The potential term is the Josephson energy of the Andreev levels, the kinetic term is the charging energy. After a simple computation of the backscattering matrix elements (the off-diagonal entries in the potential term), we arrive to the following Hamiltonian:

$$
H=H(\chi)+\frac{1}{2} E_{C}\left(\pi_{\chi}-N\right)^{2}
$$

where

$$
H(\chi)=\Delta\left(\begin{array}{cc}
-\cos \frac{\chi}{2} & r^{1 / 2} \sin \frac{\chi}{2} \\
r^{1 / 2} \sin \frac{\chi}{2} & \cos \frac{\chi}{2}
\end{array}\right)
$$

Here $\chi$ is the phase difference across the contact, $r$ is the reflection coefficient. Obviously, the eigenvalues of $H(\chi)$ reproduce the result (10). The number of Cooper pairs at the grain $\pi_{\chi}$ is the momentum conjugate to $\chi,\left[\chi, \pi_{\chi}\right]=i$. Notice that $\chi$ takes values on the circle $\chi=\chi+2 \pi$, and, accordingly, $\pi_{\chi}$ is quantized to take integer values. We may also write $\pi_{\chi}=-i \partial / \partial \chi$.

This Hamiltonian loses its validity at the top of the upper band at $\chi=2 \pi n$, where the upper Andreev state mixes with the continuous spectrum (fig. 2). Howerver, the probability of the phase $\chi$ to reach the top of the upper band of $H(\chi)$ is exponentially small at $E_{C} \ll \Delta$ (smaller than the tunneling probability). The adiabatic-diabatic crossover is determined by the properties of the system near the minimal-gap point $\chi=\pi$. Therefore, we may neglect the transitions to continuous spectrum at $\chi=2 \pi n$. At the same time, we must disregard tunneling porcesses via the top of the upper Andrees band (next-nearest-neighbor tunneling) which is present in the Hamiltonian (4)-(5), but not in the original system. The nearest-neighbor tunneling is a feature of our model and is beyond the precision of our approximation.

There are two opposite limits of the problem: small and "large" reflection.

At zero reflection, the Hamiltonian splits into lower and upper components. Within each component the potential is periodic with the period $4 \pi$. As explained above, we must neglect the next-nearest-neighbor tunneling via the top of the bands. Therefore, the potential minima of $H(\chi)$ are disconnected and cannot tunnel to each other, $\varepsilon=0$.

The opposite limit is the case of "large" reflection (the precise meaning of "large reflection" consistent with $r \ll 1$ will be clarified below). In this limit, the gap opens in the spectrum of Andreev states, and the system adiabatically follows the lower state. We can replace the two-level Hamiltonian $H(\chi)$ by its lowest eigenvalue and arrive to the 
quantum-mechanical problem of a particle in a periodic potential. The quasiclassical limit of this problem is solved in the textbook [18]. In our notation the answer reads as follows:

$$
\varepsilon_{a d}=\operatorname{const} \sqrt{E_{C} \Delta} \exp \left(-S_{c l}\right),
$$

where

$$
S_{c l}=B_{1} \sqrt{\frac{\Delta}{E_{C}}}-\frac{1}{4} \log \frac{\Delta}{E_{C}}+O(1)
$$

is the classical action connecting two nearest minima (or more precisely the two return points). The numerical constant $B_{1}$ is of order one (at $r \rightarrow 0, B_{1}=4.69+1.41 r \log r+\ldots$ ). by

To study how the adiabaticity is destroyed it is useful to introduce the dimensionless "coherence factor" $f(r)$ defined

$$
\varepsilon=f(r) \varepsilon_{a d}
$$

where $\varepsilon_{a d}$ is the amplitude of oscillations of the ground-state energy derived in the adiabatic approximation (with only the lowest Andreev state included). We see that $f(0)=0, f\left(r \gg r_{a d}\right)=1$. The crossover scale $r_{a d}$ can be derived by computing the corrections to $f(r)$ in these two limits.

First consider the limit of weak backscattering $\left(r \ll r_{a d}\right)$. In this limit we take the wavefunction to be the ground state of the Hamiltonian with zero $r$ (at a given wavevector $N$ ), and then compute the first-order correction in $r^{1 / 2}$ to the energy. The wavefunction is of "tight-binding" type and is generated by the "ground-state" wavefunctions $\Psi_{i}$ localized in the potential minima (diabatic terms). The components of the two-dimensional vectors $\Psi_{i}$ alternate:

$$
\Psi_{i}=\left(\begin{array}{c}
\Psi_{i}(\chi) \\
0
\end{array}\right), \quad \Psi_{i+1}=\left(\begin{array}{c}
0 \\
\Psi_{i+1}(\chi)
\end{array}\right) .
$$

Then we find

$$
\varepsilon=2\left\langle\Psi_{i}\left|H_{12}(\chi)\right| \Psi_{i+1}\right\rangle=2 r^{1 / 2} \Delta \int d \chi \Psi_{i}^{*}(\chi) \Psi_{i+1}(\chi) \sin \frac{\chi}{2}
$$

(We assume the wavefunctions $\Psi_{i}$ to be normalized). It is important that $\Psi_{i}$ and $\Psi_{i+1}$ are wavefunctions for different potentials $\left(-\Delta_{0} \cos (\chi / 2)\right.$ and $\left.\Delta_{0} \cos (\chi / 2)\right)$ and the overlap integral (10) has a saddle point at the minimal-gap point $\chi=\pi$, and it reduces the effective region of integration to $|\chi-\pi| \leq\left(E_{C} / \Delta\right)^{1 / 4}$. The normalization of the quasiclassical tail of the wavefunctions $\Psi_{i}(\chi)$ yields

$$
\Psi(\chi=\pi)=\exp \left(-S_{c l}(\chi=\pi)\right)
$$

(up to a numerical factor independent of $E_{C} / \Delta$ ). Thus we obtain

$$
\varepsilon \sim r^{1 / 2} \Delta\left(\frac{E_{C}}{\Delta}\right)^{1 / 4} \exp \left(-S_{c l}\right),
$$

i.e., in terms of the "coherence factor" $f(r)$,

$$
f(r) \sim r^{1 / 2}\left(\frac{\Delta}{E_{C}}\right)^{1 / 4} .
$$

The physical meaning of the integral $(10)$ is the summation over all paths shown in fig. 3a.
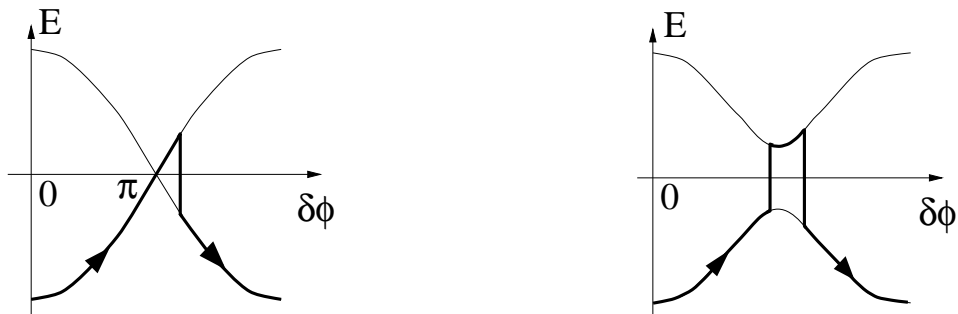

FIG. 3. Tunneling paths in the diabatic (a) and adiabatic (b) limits. These diagrams represent the lowest-order corrections to the phase tunneling amplitudes in the diabatic and adiabatic limits respectively. 
The above calculation shows that the crossover scale to adiabatic behavior is

$$
r_{a d} \sim\left(\frac{E_{C}}{\Delta}\right)^{1 / 2}
$$

In fact, we neglected the effect of change in the classical action $S_{c l}$ due to opening a gap; this effect is estimated to be of order

$$
\delta S_{c l} \sim \sqrt{\frac{\Delta}{E_{C}}} r \log r
$$

i. e. it is a higher-order effect than the change in $f(r)$ proportional to $r^{1 / 2}$. Notice that the characteristic scale of this change in the classical action is again $r_{a d} \sim \sqrt{E_{C} / \Delta}\left(\right.$ corresponding to $\left.\delta S_{c l} \sim 1\right)$.

We may alternatively find the crossover scale $r_{a d}$ by computing the lowest order correction to the "coherence factor" $f(r)$ in the adiabatic limit. In this limit the Hamiltonian (4,5) may be rewritten in adiabatic terms (the voltage $N$ is for simplicity moved to the boundary condition $\Psi(\chi+2 \pi)=e^{2 i \pi N} \Psi(\chi)$ by a gauge transformation) as

$$
H=-\frac{E_{C}}{2}\left(\frac{\partial}{\partial \chi}\right)^{2}+D(\chi)-\frac{E_{C}}{2}\left[G(\chi) \frac{\partial}{\partial \chi}+\frac{\partial}{\partial \chi} G(\chi)\right]-\frac{E_{C}}{2} G^{2}(\chi),
$$

where

$$
D(\chi)=\left(\begin{array}{cc}
E_{1}(\chi) & 0 \\
0 & E_{2}(\chi)
\end{array}\right)
$$

is the diagonalized form of the matrix (5), and

$$
G(\chi)=\left(\begin{array}{cc}
0 & g(\chi) \\
-g(\chi) & 0
\end{array}\right), \quad g(\chi)=\left\langle 0\left|\frac{\partial}{\partial \chi}\right| 1\right\rangle,
$$

and $|0\rangle$ and $|1\rangle$ are the eigenvectors of the matrix (5). The last term in the Hamiltonian (16) can be shown to give smaller corrections than the term of the first order in $G(\chi)$. A careful perturbation theory in $g(\chi)$ gives in second order

$$
1-f(r) \sim \int_{\chi_{1}<\chi_{2}} e^{S_{1}\left(\chi_{1}, \chi_{2}\right)-S_{2}\left(\chi_{1}, \chi_{2}\right)} g\left(\chi_{1}\right) g\left(\chi_{2}\right) d \chi_{1} d \chi_{2},
$$

where $S_{1,2}\left(\chi_{1}, \chi_{2}\right)$ are the classical actions along the lower and the upper adiabatic branches between the points $\chi_{1}$ and $\chi_{2}$. This integral corresponds to summation over all tunneling paths shown in fig. $3 \mathrm{~b}$. The function $g(\chi)$ for the given matrix $H(\chi)$ is a lorentzian peak at $\chi=\pi$ of height $r^{-1 / 2}$ and width $r^{1 / 2}$. Putting everything together, the integral (19) is calculated to be

$$
1-f(r) \sim \frac{1}{r} \sqrt{\frac{E_{C}}{\Delta}} .
$$

This asymptotics agrees with the found previously crossover scale (14).

To summarize the results of this section, the characteristic scale for adiabatic-diabatic crossover in a nearly-ballistic single contact is found to be $r_{a d} \sim \sqrt{E_{C} / \Delta}$. The phase tunneling amplitude is proportional to the gate-voltage modulation of the effective capacitance of the island, and thus can be directly measured. At low reflection coefficients, these oscillations are proportional to $\sqrt{r}$, like in the normal 1-channel QPC [2].

\section{ADIABATIC APPROXIMATION OF A DOUBLE-JUNCTION SYSTEM.}

Now turn to the case of a double-junction system (fig. 1b). As before, we assume that the reflection probabilities in both contacts are small, $r_{i} \ll 1$, that the charging energy $E_{C} \ll \Delta$ and that the temperature is sufficiently low to prohibit single-electron excitations on the grain. To adjust electrostatic potential of the grain we again use a gate terminal, $N=V_{g} C / 2 e$ denotes the dimensionless gate voltage, as before.

For the moment, to simplify the discussion we assume that the reflection coefficients in the contacts are greater than the crossover scale $r_{a d}$ found in the previous section and, therefore, we may consider only the lower adiabatic 
branch of the Andreev states. In fact, the results may be extended further to the case $r_{i}<r_{a d}$ by using appropriate "coherence factors" $f(r)$, similar to those in the previous section.

We set the superconducting phase on one of the leads to be zero; the phase on the other lead $\alpha$ is assumed to be fixed externally. Then the total Josephson energy of the two contacts is (fig. 4):

$$
U(\chi)=U_{1}(\chi)+U_{2}(\alpha-\chi),
$$

where

$$
U_{i}(\delta \phi)=-\Delta \sqrt{1-t_{i} \sin ^{2}(\delta \phi / 2)}
$$

are the lower adiabatic Andreev terms in the two junctions.

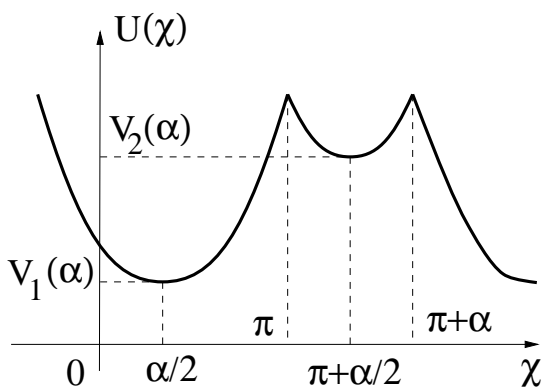

FIG. 4. Potential $U(\chi)$. At $\alpha \neq 0$ it has two minima. Finite backscattering in the contacts smoothes the summits of the potential, but leaves the bottom of the wells unchanged.

At $t_{1}=t_{2}=1$, the potential $U(\chi)$ obviously has two minima - at $\chi=\alpha / 2$ and at $\chi=\alpha / 2+\pi$ - and sharp summits at $\chi=\pi$ and $\chi=\pi+\alpha$ (fig. 4). At small nonzero $r_{i}$, gaps open at the crossing points of Andreev levels, which smoothes the summits of $U(\chi)$. Still, the bottom of the potential remains practically unchanged.

The adiabatic Hamiltonian for the double junction looks like follows:

$$
H(\alpha, N)=U(\chi)+U(\alpha-\chi)+\frac{1}{2} E_{C}\left(-i \frac{\partial}{\partial \chi}-N\right)^{2} .
$$

The potential term of the Hamiltonian is the sum of Josephson energies of the contacts, the kinetic term is the Coulomb energy of the charge at the grain.

\section{JOSEPHSON CURRENT.}

The condition $E_{C} \ll \Delta$ allows us to treat the Coulomb term in the Hamiltonian perturbatively. First, neglecting the Coulomb term, we obtain a classical system on the circle in the potential (21) with two minima. The energies of the minima are $V_{1}(\alpha)=-2 \Delta|\cos (\alpha / 4)|$ and $V_{2}(\alpha)=-2 \Delta|\sin (\alpha / 4)|$ (see fig. 4). To a very good precision, we may neglect backscattering in determining the minima - except near the point $\alpha=0$. Since all the Coulomb effects occur near the resonance point $\alpha=\pi$, this approximation is justified. At zero temperature, our classical system prefers the lowest of the minima. Thus the energy of the S-S-S system in the absence of the Coulomb term is given by

$$
E(\alpha)=-2 \Delta \cos (\alpha / 4) \quad \text { for } \quad-\pi<\alpha<\pi
$$

(see fig. 5). Differentiating this energy with respect to the phase $\alpha$ gives the Josephson current

$$
I(\alpha)=2 e \frac{\partial E(\alpha)}{\partial \alpha}=\Delta \sin \frac{\alpha}{4} \quad \text { for } \quad-\pi<\alpha<\pi
$$

(fig. 6). Notice that the current has large jumps at the points of level crossing $\alpha=\pi+2 \pi n$. Qualitatively this picture is very similar to the case of a single S-S ballistic junction, but the shape of the current-phase dependence $I(\alpha)$ is different. 


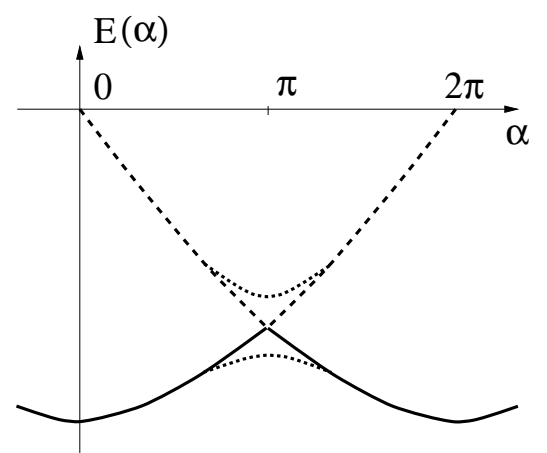

FIG. 5. Classical minimum of the potential $U(\chi)$ as a function of the external phase difference $\alpha$. Dotted line shows the quantum gap opened by the Coulomb term.

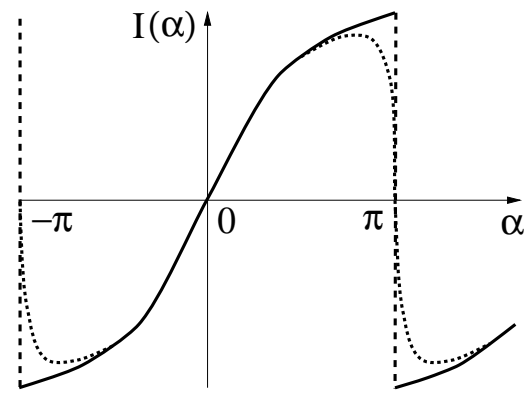

FIG. 6. Josephson current as a function of the external phase difference $\alpha$. Dotted line shows smearing of the singularity due to the Coulomb term.

If we assume a non-zero temperature $T \ll \Delta$, the occupation of the upper minimum is exponentially small except in the vicinity of the level-crossing point $|\alpha-\pi| \sim T / \Delta$. Thus, the effect of the temperature results in the smearing of the singularity in $I(\alpha)$ at $\alpha=\pi$.

Another source of level mixing near the singular point $\alpha=\pi$ is quantum fluctuations, i.e. the fluctuations arising from the kinetic term in the Hamiltonian (23). They result in nonzero amplitudes of tunneling through the two potential barriers between the potential minima. Due to the shift in the "angular momentum" by $N$, the wave functions in the two potential wells aquire an additional factor $\exp (i N \chi)$. This results in the relative phase of the two tunneling amplitudes by $2 \pi N$. The net tunneling amplitude (defining the level splitting) may be written as

$$
H_{12}(N) \equiv \Delta \gamma(N)=\Delta\left(\gamma_{1} e^{i \pi N}+\gamma_{2}^{-i \pi N}\right)
$$

where $\gamma_{1}$ and $\gamma_{2}$ are the two amplitudes of phase tunneling in the two different directions (i.e. of phase slip processes in the two different contacts). Below we assume that these amplitudes are computed at the level-crossing point $\alpha=\pi$, where they are responsible for level splitting.

The amplitudes $\gamma_{1}$ and $\gamma_{2}$ obey all asymptotics derived in the previous section (except for numerical factors). When the backscattering in the contacts $r \gg r_{a d}$, they may be found in the quasiclassical approximation:

$$
\gamma_{1,2} \sim\left(\frac{E_{C}}{\Delta}\right)^{1 / 4} \exp \left(-B_{2} \sqrt{\frac{\Delta}{E_{C}}}\right) \ll 1
$$

where $B_{2} \sim 1$ is determined by the classical action connecting the two potential minima (at $r \ll 1, B_{2} \cong 1.45+$ $2.20 r \log r+\ldots)$. At $r \ll r_{a d}$, the tunneling amplitudes are

$$
\gamma_{1,2} \sim r^{1 / 2} \exp \left(-B_{2} \sqrt{\frac{\Delta}{E_{C}}}\right)
$$

For the best observation of Coulomb oscillations, $\gamma_{1}$ and $\gamma_{2}$ must be of the same order, but not very small. In the ideal case $\gamma_{1}=\gamma_{2}=\gamma$ the total amplitude

$$
\gamma(N)=2 \gamma \cos (\pi N)
$$


Although the periodic dependence (29) has $4 e$ period as function of the "external charge" $Q_{x}=C V_{g} \equiv 2 e N$, the Josephson current and its fluctuations depend on $|\gamma(N)|^{2}$ only (cf. eqs.(32.34) below), and their period is $2 e$ as expected [3].

The characteristic scale for the $r$-dependence of $B_{2}$ is $\delta r \sim \sqrt{E_{C} / \Delta}$, therefore for $\gamma_{1}$ and $\gamma_{2}$ to be of the same order, the transparencies of the two contacts must differ by no more than $\left|r_{1}-r_{2}\right| \leq \sqrt{E_{C} / \Delta}$.

Here we should comment on the difference of our result (26)-(28) from the normal two-channel system discussed in [2]. In the normal system the two tunneling amplitudes multiply, and the net ground-state energy oscillations are proportional to $r \ln r$ at small $r$. In the superconducting system, the external leads have different superconducting phases, and the tunneling in the two contacts occurs at different values of the phase on the grain. Therefore, the tunneling amplitudes add with some phase factors and give the asymptotic of $\sqrt{r}$ at $r \rightarrow 0$. In fact, the oscillations in the superconducting system will be proportional to $r$ (similarly to the normal system [2]) in a different limit — at the phase difference $\alpha=0$, when the potential $U(\chi)$ has a single minimum and a single barrier.

The hybridized energy levels in the vicinity of $\alpha=\pi$ are given by the eigenvalues of the $2 \times 2$ Hamiltonian

$$
H(\alpha, N)=\left(\begin{array}{cc}
V_{1}(\alpha) & H_{12}(N) \\
H_{12}(N) & V_{2}(\alpha)
\end{array}\right) .
$$

Diagonalization gives the two energy levels:

$$
E_{1,2}(\alpha, N)=-\Delta\left[\left|\sin \frac{\alpha}{4}\right|+\left|\cos \frac{\alpha}{4}\right| \pm \sqrt{\left(\left|\sin \frac{\alpha}{4}\right|-\left|\cos \frac{\alpha}{4}\right|\right)^{2}+\gamma^{2}(N)}\right],
$$

the off-diagonal matrix elements of the Hamiltonian open a gap at the level-crossing point $\alpha=\pi$ (fig. 5). This gap periodically depends on the gate voltage $V_{g}$, and these oscillations comprise the Coulomb effects in the S-S-S junction.

We can obtain the Josephson current by differentiating the energy levels with respect to the phase $\alpha$. The gap results in smearing the singularity in $I(\alpha)$ even at zero temperature (fig. 6):

$$
I(\alpha)=\frac{\Delta}{\sqrt{2}} \sin \left(\frac{\alpha-\pi}{4}\right)\left[1-\frac{\cos \left(\frac{\alpha-\pi}{4}\right)}{\sqrt{\sin ^{2}\left(\frac{\alpha-\pi}{4}\right)+\frac{1}{2} \gamma^{2}(N)}}\right] \quad \text { for } \quad \alpha \sim \pi .
$$

The width of the crossover at $\alpha=\pi$ depends periodically on $V_{g}:|\alpha-\pi| \sim|\gamma(N)|$.

In the above discussion we neglected the excited oscillator states. The interlevel spacing for the excitations in the potential wells is of order $\sqrt{\Delta E_{C}} \gg \Delta \gamma$. Therefore the Coulomb effects have a much smaller energy scale and the excited states do not participate in mixing the ground states of the two potential wells.

At a nonzero temperature these Coulomb effects will compete with the smearing by temperature so that the width of the singularity at $\alpha=\pi$ is given at nonzero temperature $T \ll \Delta$ by $|\alpha-\pi| \sim \max (\gamma(N), T / \Delta)$. Therefore, in order for Coulomb effects to dominate the thermal fluctuations, we must have $T \leq \gamma \Delta$.

It is instructive to compare this picture with the case of multi-channel tunnel S-S-S junction (to distinguish from the results of [3] we should remark that we consider the opposite to their assumption $\Delta<E_{C}$ limit). If we develop a similar theory for tunnel Josephson junctions, we find that the potentials (21), (22) are both sinusoidal, and, therefore, the total potential (21) has only one minimum (versus two in the nearly ballistic system). In the tunnel S-S-S system the current-phase relation $I(\alpha)$ has a smearing at $\alpha=\pi$ due to the difference between the critical currents of the two Josephson contacts. The Coulomb effects compete with this smearing and in order to win, the charging energy $E_{C}$ must be greater than the difference of the critical currents. In the tunnel system the corresponding splitting $\gamma$ is linear in $E_{C}$ while in the nearly ballistic system it is exponentially small. Otherwise, Coulomb oscillations in $I(\alpha)$ will appear similar in these two cases.

To summarize this section, we observed that the Coulomb effects in the one-channel S-S-S junction smears the singularity in the Josephson current $I(\alpha)$ at the critical value $\alpha=(2 n+1) \pi$. This smearing depends periodically on the potential of the grain with the period $2 e / C$ and is exponentially small in the adiabatic parameter $E_{C} / \Delta \ll 1$. The smearing is the result of mixing the two states in the potential minima of the Josephson energy.

\section{FLUCTUATIONS OF THE JOSEPHSON CURRENT.}

In this section we compute the low-frequency spectrum of the fluctuations of the Josephson current in our model. We shall be interested in frequencies much less than the oscillator energy scale $\sqrt{\Delta E_{C}}$, thus we consider only transitions 
between the eigenstates of the reduced ground-state Hamiltonian (30). We also assume that the temperature is lower than $\sqrt{\Delta E_{C}}$, then we may disregard the excited oscillator states and the internal noise in the contacts (discussed in [12 14, 19]). Obviously, under these assumptions we can observe current fluctuations only in the close vicinity of the resonance point $\alpha= \pm \pi$, where the energies (31) of the two low-lying states are close to each other.

We expect to observe two peaks in the noise spectrum - one at zero frequency (due to the thermal excitations above the ground state), and the other at the transition frequency $\left|E_{1}-E_{2}\right|$ (from the off-diagonal matrix elements of the current operator). In this section we compute the intergal weights of these peaks and postpone the discussion of their width (determined by dissipative processes) until elsewhere.

Discuss first the zero-frequency peak. In our approximation it is just the thermal noise of a two-level system. In the vicinity of the resonance point $\alpha=\pi$ we can linearize the spectrum $V_{1,2}(\alpha)$ and make an approximation that one of the two states carries the current $I(\alpha, N)$, and the other $-I(\alpha, N)$. The spectral weight of the noise is then given by a simple formula:

$$
S_{0}(\alpha, N, T) \equiv\left\langle I^{2}\right\rangle-\langle I\rangle^{2}=\frac{I^{2}(\alpha, N)}{\cosh ^{2} \frac{E_{1}-E_{2}}{2 T}}
$$

Substituting $I(\alpha, N)$ and $E_{1,2}(\alpha, N)$ from the previous section, we obtain the noise intensity near the resonance:

$$
S_{0}(\alpha, N, T)=\frac{\Delta^{2}}{2} \frac{\left(\frac{\alpha-\pi}{2 \sqrt{2}}\right)^{2}}{\left(\frac{\alpha-\pi}{2 \sqrt{2}}\right)^{2}+\gamma^{2}(N)} \cosh ^{-2}\left(\frac{\Delta}{T} \sqrt{\left(\frac{\alpha-\pi}{2 \sqrt{2}}\right)^{2}+\gamma^{2}(N)}\right) .
$$

For the effect of the Coulomb interaction to be observable, the temperature must be smaller than the Coulomb gap: $T \leq \gamma \Delta$. At constant $T$ and $N$, the noise decreases exponentially as $\alpha$ goes away from its critical value $\alpha=\pi$, and at $\alpha=\pi$ the noise is suppressed in the interval $|\alpha-\pi|<\gamma(N)$ (fig. 7). Interplay between these two factors results in the strong dependence of the peak value of the noise on the potential of the grain. The peak value of the noise $\max _{\alpha} S(\alpha, N, T)$ is plotted against $N$ in fig. 8. Most favorable is the case of identical contacts, when $\gamma_{1}=\gamma_{2}=\gamma$ and, therefore, $\gamma(N)=2 \gamma \cos (\pi N)$. In this case, when $\cos (\pi N) \ll T / \gamma \Delta$ (small gap limit) the noise takes its maximal value $S \approx \Delta^{2} / 2$. In the opposite limit of large gap $(\cos (\pi N) \gg T / \gamma \Delta)$ the noise decreases exponentially: $S \approx \Delta^{2}\left[\frac{T}{\Delta \gamma|\cos \pi N|} \exp \left(-4 \frac{\Delta \gamma|\cos \pi N|}{T}\right)\right]$. The noise has a sharp peak at the resonance point $\cos \pi N=0$, where two levels on the grain with different electron numbers have equal energies.

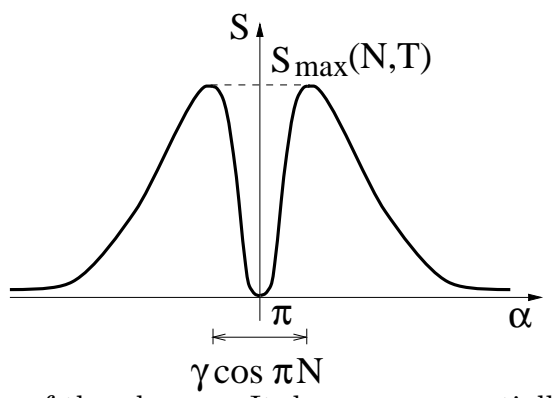

FIG. 7. Zero frequency noise as a function of the phase $\alpha$. It decays exponentially for $\alpha$ far from the resonance point $\alpha=\pi$. At the very resonance point, the noise is suppressed, because both of the two states carry nearly zero Josephson current.

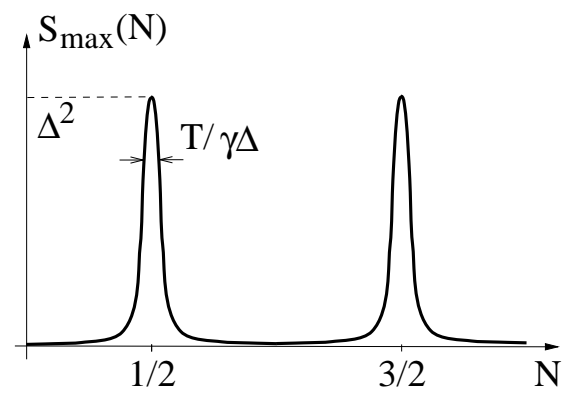

FIG. 8. Maximal value of the noise versus the potential of the grain. The period of the peaks corresponds to the period $2 e$ of the induced charge $Q=C V_{g}$. The width of the peaks depends on the capacity of the grain. 
Now turn to the noise peak at the interlevel frequency $\omega=\left|E_{1}-E_{2}\right|$. Since now $\omega$ can be large compared to $T$, one needs to discern between different kinds of frequency-dependent correlation functions, which can be measured as a noise intensity in different experimental situations [21]; here we mean by noise the Fourrier spectrum of the time-symmetric current-current correlation function. In our approximation of a two-level system such a noise is temperature independent, and its weight is determined purely by the off-diagonal matrix element:

$$
S_{\omega}=\frac{1}{2}|\langle 1|I| 2\rangle|^{2} .
$$

A straightforward computation for the Hamiltonian (30) and $I=2 e(\partial H / \partial \alpha)$ gives (in the vicinity of $\alpha=\pi)$ :

$$
\langle 1|I| 2\rangle=\frac{\Delta^{2} \gamma(N)}{\omega}\left(\cos \frac{\alpha}{4}+\sin \frac{\alpha}{4}\right)
$$

and

$$
S_{\omega}(\alpha, N)=\Delta^{2}\left(\frac{\Delta \gamma(N)}{\omega}\right)^{2} \cos ^{2} \frac{\alpha-\pi}{4}
$$

This result contrasts the corresponding noise intensity in the single quantum point contact (found in [12, 19, 13]). In the single quantum point contact the correponding noise intensity $S_{\omega}$ is temperature-dependent, because that system has four possible states (or, alternatively, two fermion levels). In the case of the double junction the system has only two states differing by the phase on the grain, and the quantum fluctuations $S_{\omega}$ become temperature-independent.

\section{CONCLUSIONS}

We have developed a theory of Coulomb oscillations of the Josephson current and its noise power via the S-S-S system with nearly ballistic quantum point contacts. The period of Coulomb oscillations as function of the gate potential is $V_{g}^{0}=2 e / C$. These oscillations arise from the quasiclassical tunneling of the superconducting phase on the grain and are, therefore, exponentially small in $\sqrt{E_{C} / \Delta}$ at $E_{C} \ll \Delta$. In addition, we predict a crossover from adiabatic to diabatic tunneling at the backscattering probability $r_{a d} \sim \sqrt{E_{C} / \Delta}$. At backscattering below $r_{a d}$, the amplitude $\varepsilon$ of the Coulomb oscillations is proportional to the square root of the smallest (of the two contacts) reflection probability $\sqrt{r_{\min }}$. This constrasts the case of a normal double-contact system [20] where $\varepsilon$ is proportional to the product $\sqrt{r_{1} r_{2}}$.

The average Josephson current-phase relation $I(\alpha)$ is shown to be strongly non-sinusoidal and roughly similar to the one known for a single nearly ballistic QPC, in the sense that it contains sharp "switching" between positive and negative values of the current as the phase varies via $\alpha=\pi$. The new feature of our system is that it is possible to vary the width of the swithching region $\delta \alpha$ by the electric gate potential $V_{g}$; in the case of equal reflection probabilities $r_{1}=r_{2}$ this electric modulation is especially pronounced, $\delta \alpha \propto\left|\cos \left(\pi C V_{g} / 2 e\right)\right|$. The noise spectrum of the supercurrent is found to consist mainly of two peaks: the "zero-frequency" peak due to rare thermal exitations of the upper level of the system, and another one centered around the energy difference $\omega_{\alpha}$ between the two levels. The widths of these peaks are determined by the inverse life-time $\tau$ of the two states of our TLS, which is due to electron-phonon and electromagnetic couplings. Both these sources of level decay are expected to be very weak in the system considered, but the corresponding quantitative analysis is postponed for the future studies, so we present here only the results for the frequency-integrated (over those narrow intervals $\sim 1 / \tau$ ) noise power.

The S-S-S device with almost ballistic contacts is a new type of a system which may be used as a realization of an artificial "spin 1/2" - an elementary unit for quantum computations. In comparison with usual Josephson systems with tunnel junctions which were proposed for the use in adiabatic quantum computatons [11], the advantage of our system is that it may operate at considerably higher values of the Josephson critical currents; moreover, the currentphase characteristics of such a system is almost universal in the sense that it is determined mainly by the microscopic parameters of the SC materials and only weakly depends on the specifics of contact fabrication.

We are grateful to K. A. Matveev, Yu. V. Nazarov and especially to G. B. Lesovik for many useful discussions. This research of M.V.F. was supported by the INTAS-RFBR grant \# 95-0302, the collaboration grant \# 7SUP J048531 from the Swiss National Science Foundation and the DGA grant \# 94-1189. 
[1] L. I. Glazman and K. A. Matveev, ZhETF 98 (1990), 1834 [Sov. Phys. JETP 71 (1990) 1031]

[2] K. A. Matveev, Phys. Rev. B 51 (1995), 1743 [e-print cond-mat/9412029

[3] L. I. Glazman, K. A. Matveev et al., Physica B 203 (1994), 316

[4] H. Takayanagi, T. Akazaki and J. Nitta, Phys. Rev. Lett. 75 (1995), 3533

[5] C. W. Beenakker and H. van Houten, Phys. Rev. Lett. 66 (1991), 3056

[6] M. C. Koops et al., Phys. Rev. Lett. 77 (1996), 2542

[7] A. Yu. Kasumov et al, Phys. Rev. Lett. 77 (1996), 3029

[8] A. Yu. Kitaev, "Fault-tolerant quantum computations by anyons", e-print quant-ph/9707021

[9] D. P. Divincenzo, "Topics in quantum computers", e-print cond-mat/9612126.

[10] A. Shnirman, G. Schoen and Z. Hermon, "Quantum manipulations of small Josephson junctions", e-print condmat/9706016.

[11] D. V. Averin, "Adiabatic quantum computations with Cooper pairs", e-print quant-ph/9706026.

[12] A. Martin-Rodero, A. Levy Yeyati and F. J. Garcia-Vidal, Phys. Rev. B 53 (1996), R8891

[13] D. Averin and H. T. Imam, Phys. Rev. Lett. 76 (1996), 3814 [e-print cond-mat/9511128]

[14] G. B. Lesovik and A. Golubov, in Proc. of the 31 Recontre de Moriond, Eds. T. Martin et al (1996).

[15] C. W. Beenakker, Phys. Rev. Lett. 67 (1991), 3836

[16] W. Lichten, in "Atomic Physics", Vol.4, Eds. G. zu Putlitz et al (Plenum, New York, 1975)

[17] A. I. Larkin and Yu. N. Ovchinnikov, Phys. Rev. B 28 (1983), 6281

[18] L. D. Landau and E. M. Lifshits, "Quantum Mechanics: non-relativistic theory", Pergamon Press, Oxford, 1977

[19] A. Martin-Rodero et al., "Comment on 'Supercurrent Noise in Quantum Point Contacts", e-print cond-mat/9607079

[20] A. Furusaki and K. A. Matveev, Phys. Rev. B 52 (1995), 16676

[21] G. B. Lesovik, R. Loosen, Pis'ma ZhETF 65 (1997), 280; G. B. Lesovik, in Proceedings of the Conference "Meso-97", Chernogolovka; to be published in Usp.Fiz.Nauk (1998). 